Iternational Electronic Journal of Algebra

VOLUME 29 (2021) 107-119

DOI: $10.24330 /$ ieja.852029

\title{
MODULES FOR WHICH EVERY ENDOMORPHISM HAS A NON-TRIVIAL INVARIANT SUBMODULE
}

\author{
Mohamed Benslimane, Hanane EL Cuera and Rachid Tribak \\ Received: 26 January 2020; Revised: 20 June 2020; Accepted: 25 June 2020 \\ Communicated by Christian Lomp
}

\begin{abstract}
All rings are commutative. Let $M$ be a module. We introduce the property $(\mathbf{P})$ : Every endomorphism of $M$ has a non-trivial invariant submodule. We determine the structure of all vector spaces having $(\mathbf{P})$ over any field and all semisimple modules satisfying $(\mathbf{P})$ over any ring. Also, we provide a structure theorem for abelian groups having this property. We conclude the paper by characterizing the class of rings for which every module satisfies $(\mathbf{P})$ as that of the rings $R$ for which $R / \mathfrak{m}$ is an algebraically closed field for every maximal ideal $\mathfrak{m}$ of $R$.
\end{abstract}

Mathematics Subject Classification (2020): 12E05, 12F99, 13C05, 13C99, 15A04, 15A18, 15B99

Keywords: Algebraically closed field, characteristic polynomial of a matrix (an endomorphism), fully invariant submodule, homogeneous semisimple module, invariant submodule

\section{Introduction}

The notion studied in this article has its roots in operator theory. Let $\mathcal{B}(\mathcal{H})$ be the algebra of all bounded linear operators on a separable infinite dimensional complex Hilbert space $\mathcal{H}$. A closed subspace $M$ of $\mathcal{H}$ is said to be a non-trivial invariant subspace for $T \in \mathcal{B}(\mathcal{H})$ if $M \neq 0, M \neq \mathcal{H}$ and $T(M) \subseteq M$. The invariant subspace problem can be stated as follows:

Does every bounded linear operator $T \in \mathcal{B}(\mathcal{H})$ have a non-trivial invariant closed subspace?

A number of research papers have been devoted to the study of this conjecture which is still open. Following [1], the research on this problem was initiated by J. von Neumann who proved in the early thirties of the last century that every linear compact operator on a Hilbert space has a non-trivial invariant closed subspace. The proof of this result was never published. Later in 1954, Aronszajn and Smith [1] extended von Neumann's result to the Banach spaces setting. In 1966 [2], Bernstein and Robinson proved that every polynomially compact operator $T$ on a 
Hilbert space (i.e., $P(T)$ is compact for some nonzero polynomial $P$ ) has a nontrivial invariant subspace. In 1973 [5], Lomonosov showed that every bounded linear operator on a complex Banach space which commutes with a nonzero compact operator has a non-trivial invariant closed subspace. Further details about the developments on the above conjecture can be found in [4].

In this paper, we examine this problem from an algebraic point of view by extending it to a module theoretic version. Let $R$ be a commutative ring and let $M$ be an $R$-module. A submodule $N$ of $M$ is said to be invariant under an $R$-endomorphism $f$ of $M$ if $f(N) \subseteq N$. The module $M$ is said to have property $(\mathbf{P})$ if every $R$-endomorphism $f$ of $M$ has a non-trivial invariant submodule. The focus of our investigations is to explore and study modules satisfying $(\mathbf{P})$.

In Section 2, we prove that every infinitely generated semisimple module has $(\mathbf{P})$ (Proposition 2.12). It is shown that for a commutative field $K$, a $K$-vector space $V$ with $\operatorname{dim}(V)=n \geq 2$ satisfies $(\mathbf{P})$ if and only if every monic polynomial $P(X) \in$ $K[X]$ of degree $n$ is reducible (Theorem 2.6). Also, we determine the structure of abelian groups having $(\mathbf{P})$ (Theorem 2.21). Some examples are provided to show that even a semisimple module needs not have $(\mathbf{P})$, in general.

In the main result of Section 3, we characterize the class of rings $R$ for which every nonzero finitely generated $R$-module which is not simple has $(\mathbf{P})$. It turns out that this class of rings is precisely that of rings $R$ for which $R / \mathfrak{m}$ is an algebraically closed field for every maximal ideal $\mathfrak{m}$ of $R$ (Theorem 3.4).

Throughout this article, all rings are commutative with identity and all modules are unital. Let $R$ be a ring and let $M$ be an $R$-module. A submodule $L$ of $M$ is called non-trivial if $L \neq 0$ and $L \neq M$. We use $\operatorname{Rad}(M), \operatorname{Soc}(M)$, and $\operatorname{End}_{R}(M)$ to denote the radical, the socle, and the endomorphism ring of $M$, respectively. The notation $N \subseteq M$ means that $N$ is a subset of $M$ and the notation $N \leq M$ means that $N$ is a submodule of $M$. By $\mathbb{Q}, \mathbb{Z}$, and $\mathbb{N}$ we denote the ring of rational numbers, the ring of integer numbers, and the set of natural numbers, respectively.

\section{Modules having (P)}

Proposition 2.1. The following are equivalent for a module $M$ :

(i) Every endomorphism of $M$ has a non-trivial invariant submodule (i.e., $\forall f \in$ $\operatorname{End}_{R}(M), \exists 0 \neq N \leq M$ such that $N \neq M$ and $\left.f(N) \subseteq N\right)$;

(ii) Every automorphism of $M$ has a non-trivial invariant submodule.

Proof. (i) $\Rightarrow$ (ii) This is immediate. 
(ii) $\Rightarrow$ (i) Let $f$ be a nonzero endomorphism of $M$ which is not an automorphism. Then $\operatorname{Kerf} \neq 0$ or $\operatorname{Imf} \neq M$. Note that $\operatorname{Kerf} \neq M$ and $\operatorname{Imf} \neq 0$. If $\operatorname{Kerf} \neq 0$, then $\operatorname{Kerf}$ is a non-trivial invariant submodule under $f$. If $\operatorname{Imf} \neq M$, then $\operatorname{Imf}$ is a non-trivial invariant submodule under $f$.

Definition 2.2. A module $M$ is said to have property $(\mathbf{P})$ if it satisfies any of the equivalent two conditions in Proposition 2.1.

Recall that a submodule $N$ of a module $M$ is called fully invariant if $f(N) \subseteq N$ for every endomorphism $f$ of $M$. It is well known that for any module $M, \operatorname{Soc}(M)$ and $\operatorname{Rad}(M)$ are fully invariant submodules of $M$. In [6], the authors studied $d u o$ modules (i.e., modules in which every submodule is fully invariant). It is clear that every nonzero duo module which is not simple satisfies $(\mathbf{P})$. So for every commutative ring $R$ which is not a field, the $R$-module $R$ has $(\mathbf{P})$.

Example 2.3. (i) It is clear that every module having a non-trivial fully invariant submodule has $(\mathbf{P})$. In particular, every module $M$ with non-trivial radical or non-trivial socle has $(\mathbf{P})$.

(ii) Let $M$ be an artinian module which is not semisimple. Then $\operatorname{Soc}(M) \neq M$. Moreover, it is well known that $\operatorname{Soc}(M) \neq 0$. Hence $M$ has $(\mathbf{P})$.

To explore modules having $(\mathbf{P})$, it is natural to begin by investigating vector spaces over a field and semisimple modules.

Proposition 2.4. Let $K$ be a field. Every infinite-dimensional $K$-vector space has $(\mathbf{P})$.

Proof. Let $V$ be a $K$-vector space of infinite dimension and let $T$ be an automorphism of $V$. Suppose that the only invariant subspaces of $V$ under $T$ are 0 and $V$. Let $0 \neq u \in V$ and consider the nonzero subspace $W$ of $V$ generated by the family $\left\{T^{k}(u), k \geq 1\right\}$. Clearly, $T(W) \subseteq W$. Therefore $W=V$ and hence $u \in W$. So there exists $p \geq 1$ such that $u=\alpha_{1} T(u)+\alpha_{2} T^{2}(u)+\cdots+\alpha_{p} T^{p}(u)$, where $\alpha_{1}, \alpha_{2}, \ldots, \alpha_{p} \in K$ and $\alpha_{p} \neq 0$. It follows that $T^{p}(u)$ belongs to the nonzero subspace $L$ of $V$ generated by the family $\left\{u, T(u), \ldots, T^{p-1}(u)\right\}$. This implies that $T(L) \subseteq L$. Note that $L$ is a finitely generated subspace of $V$. Thus $L \neq V$. This is a contradiction. Consequently, $V$ contains a non-trivial subspace which is invariant under $T$.

Next, we characterize finite-dimensional vector spaces having $(\mathbf{P})$. We begin with the following well known remark which is included for completeness. 
Remark 2.5. Let $K$ be a field and let $P(X)=X^{n}+a_{n-1} X^{n-1}+\cdots+a_{1} X+a_{0} \in$ $K[X]$ be a polynomial of degree $n \geq 2$. The companion matrix of the polynomial $P(X)$ is the $n \times n$ matrix

$$
M=\left[\begin{array}{ccccc}
0 & 1 & 0 & \cdots & 0 \\
\vdots & \ddots & \ddots & \ddots & \vdots \\
\vdots & & \ddots & 1 & 0 \\
0 & \cdots & \cdots & 0 & 1 \\
-a_{0} & -a_{1} & \cdots & \cdots & -a_{n-1}
\end{array}\right] \in M_{n}(K)
$$

Let $T$ be an endomorphism of $K^{n}$ such that $M$ is the matrix of $T$ with respect to the standard basis. It is easy to check that the characteristic polynomial of $T$ is $(-1)^{n} P(X)$.

Theorem 2.6. Let $K$ be a field and let $n \geq 2$ be an integer. Then the following statements are equivalent:

(i) The $K$-vector space $U=K^{n}$ has $(\mathbf{P})$;

(ii) Every monic polynomial $P(X) \in K[X]$ of degree $n$ is reducible.

Proof. (i) $\Rightarrow$ (ii) Assume that $U$ has $(\mathbf{P})$. Let $P(X)$ be a monic polynomial in $K[X]$ of degree $n$. By the preceding remark, there exists a nonzero endomorphism $f$ of $U$ such that the characteristic polynomial of $f$ is $(-1)^{n} P(X)$. Then $U$ contains a non-trivial subspace $V$ such that $f(V) \subseteq V$. Set $h=\operatorname{dim}(V)$. Note that $1 \leq$ $h \leq n-1$. Moreover, there exists a subspace $W$ of $V$ such that $U=V \oplus W$ and $\operatorname{dim}(W)=n-h$. Let $\mathcal{B}_{1}=\left\{e_{1}, e_{2}, \ldots, e_{h}\right\}$ be a basis for $V$ and let $g$ be the restriction of $f$ to $V$. We denote by $A_{1}$ the matrix of $g$ with respect to the basis $\mathcal{B}_{1}$. Let $\left\{e_{h+1}, \ldots, e_{n}\right\}$ be a basis for $W$. Then $\mathcal{B}_{2}=\left\{e_{1}, e_{2}, \ldots, e_{n}\right\}$ is a basis for $U$. It is easily seen that the matrix $A$ of $f$ with respect to the basis $\mathcal{B}$ has the form

$$
A=\left[\begin{array}{cc}
A_{1} & A_{2} \\
0 & A_{3}
\end{array}\right] .
$$

Let $P_{A}(X)$ and $P_{A_{i}}(X)(i \in\{1,3\})$ be the characteristic polynomials of the matrices $A$ and $A_{i}(i \in\{1,3\})$, respectively. Then $P_{A}(X)=P_{A_{1}}(X) P_{A_{3}}(X)$. It follows that $(-1)^{n} P(X)=P_{A_{1}}(X) P_{A_{3}}(X)$. That is, $P(X)=\left((-1)^{n} P_{A_{1}}(X)\right) P_{A_{3}}(X)$. Note that $\operatorname{deg}\left(P_{A_{i}}(X)\right) \geq 1$ for each $i \in\{1,3\}$.

(ii) $\Rightarrow$ (i) Let $T$ be an automorphism of $U$ and let $P(X)$ be the characteristic polynomial of $T$. We denote by $A=\left(\alpha_{i j}\right)_{1 \leq i, j \leq n}$ the matrix of $T$ with respect to the standard basis. By (ii), there exists a monic irreducible polynomial $Q(X)$ which divides $P(X)$ such that $q=\operatorname{deg}(Q(X)) \neq n$. It is well known that $K$ has an 
extension field $L$ (which is isomorphic to $K[X] /\langle Q(X)\rangle$ ) such that $[L: K]=q$ and $Q(X)$ has a root $\lambda$ in $L$. Let $\left\{\sigma_{1}, \sigma_{2}, \ldots, \sigma_{q}\right\}$ be a basis of the $K$-vector space $L$. For all $i, j$ in $\{1, \ldots, q\}$, there exist $\gamma_{i j}^{t} \in K(1 \leq t \leq q)$ such that $\sigma_{i} \sigma_{j}=\sum_{t=1}^{q} \gamma_{i j}^{t} \sigma_{t}$. Also, there exist $\lambda_{i} \in K(1 \leq i \leq q)$ such that $\lambda=\sum_{i=1}^{q} \lambda_{i} \sigma_{i}$. Since $\lambda$ is a root of $P(X)$ in $L$, there exists $0 \neq v=\left[\begin{array}{c}b_{1} \\ \vdots \\ b_{n}\end{array}\right] \in V=L^{n}$ such that $A v=\lambda v$. Note that

for every $i \in\{1, \ldots, n\}$, there exist $\beta_{i s} \in K(1 \leq s \leq q)$ such that $b_{i}=\sum_{s=1}^{q} \beta_{i s} \sigma_{s}$. Fix $l \in\{1, \ldots, n\}$. We have $\sum_{j=1}^{n} \alpha_{l j} b_{j}=\lambda b_{l}$. Hence,

$$
\sum_{j=1}^{n} \alpha_{l j}\left(\sum_{s=1}^{q} \beta_{j s} \sigma_{s}\right)=\lambda \sum_{j=1}^{q} \beta_{l j} \sigma_{j}
$$

That is,

$$
\sum_{s=1}^{q}\left(\sum_{j=1}^{n} \alpha_{l j} \beta_{j s}\right) \sigma_{s}=\sum_{i=1}^{q} \sum_{j=1}^{q} \lambda_{i} \beta_{l j}\left(\sigma_{i} \sigma_{j}\right)
$$

Therefore,

$$
\sum_{s=1}^{q}\left(\sum_{j=1}^{n} \alpha_{l j} \beta_{j s}\right) \sigma_{s}=\sum_{i=1}^{q} \sum_{j=1}^{q} \lambda_{i} \beta_{l j}\left(\sum_{s=1}^{q} \gamma_{i j}^{s} \sigma_{s}\right)
$$

i.e.,

$$
\sum_{s=1}^{q}\left(\sum_{j=1}^{n} \alpha_{l j} \beta_{j s}\right) \sigma_{s}=\sum_{s=1}^{q}\left(\sum_{j=1}^{q}\left(\sum_{i=1}^{q} \lambda_{i} \gamma_{i j}^{s}\right) \beta_{l j}\right) \sigma_{s}
$$

It follows that

$$
\sum_{j=1}^{n} \alpha_{l j} \beta_{j s}=\sum_{j=1}^{q}\left(\sum_{i=1}^{q} \lambda_{i} \gamma_{i j}^{s}\right) \beta_{l j} \text { for every } s \in\{1, \ldots, q\}
$$

For every $j, s$ in $\{1, \ldots, q\}$, set $u_{j}=\left[\begin{array}{c}\beta_{1 j} \\ \vdots \\ \beta_{n j}\end{array}\right] \in U=K^{n}$ and $\varepsilon_{j s}=\sum_{i=1}^{q} \lambda_{i} \gamma_{i j}^{s} \in K$.

A trivial verification shows that $A u_{s}=\sum_{j=1}^{q} \varepsilon_{j s} u_{j}$ for all $s \in\{1, \ldots, q\}$. This implies that the $K$-subspace $H=\left\langle u_{1}, u_{2}, \ldots, u_{q}\right\rangle$ of $U$ generated by $\left\{u_{1}, u_{2}, \ldots, u_{q}\right\}$ is invariant under $T$. This completes the proof.

To visualize the proof of the previous theorem, we provide the following example.

Example 2.7. Let $\mathbb{R}$ denote the field of real numbers. Consider the $\mathbb{R}$-vector space $U=\mathbb{R}^{4}$ and the polynomial $P(X)=\left(X^{2}+1\right)^{2} \in \mathbb{R}[X]$. Then the companion 
matrix of $P(X)$ is

$$
A=\left[\begin{array}{rrrr}
0 & 1 & 0 & 0 \\
0 & 0 & 1 & 0 \\
0 & 0 & 0 & 1 \\
-1 & 0 & -2 & 0
\end{array}\right]
$$

It is clear that $P(X)$ est divisible by $Q(X)=X^{2}+1$. Moreover, it is well known that the field $\mathbb{C}$ of complex numbers is an extension field of $\mathbb{R}$ such that $[\mathbb{C}: \mathbb{R}]=$ $2=\operatorname{deg}(Q(X))$. So, if we regard $A$ as a matrix over $\mathbb{C}$ then $A$ has two complex eigenvalues, namely $i$ and $-i$, and $v=\left[\begin{array}{r}i \\ -1 \\ -i \\ 1\end{array}\right]$ is an eigenvector of $A$ corresponding to the eigenvalue $i$. With the notation of the proof of Theorem 2.6, the coefficients $\beta_{i j}$ have the values $\beta_{11}=0, \beta_{21}=-1, \beta_{31}=0, \beta_{41}=1, \beta_{12}=1, \beta_{22}=0$, $\beta_{32}=-1$ and $\beta_{42}=0$ so that the vectors $v_{1}=\left[\begin{array}{r}0 \\ -1 \\ 0 \\ 1\end{array}\right]$ and $v_{2}=\left[\begin{array}{r}1 \\ 0 \\ -1 \\ 0\end{array}\right]$ generate a subspace of $U$ that is invariant under $A$.

Corollary 2.8. Let $K$ be a finite field and let $n \geq 2$ be an integer. Then the $K$-vector space $K^{n}$ never has $(\mathbf{P})$.

Proof. Since $K$ is finite, there exists an irreducible polynomial $Q(X) \in K[X]$ with $\operatorname{deg}(Q(X))=n$ (see [3, Corollary 2.11]). From Theorem 2.6, we deduce that the $K$-vector space $K^{n}$ does not have $(\mathbf{P})$.

Proposition 2.9. Let $K$ be a field and let $n$ be an integer with $n \geq 2$. Then the following are equivalent:

(i) Every $K$-vector space of dimension $t(2 \leq t \leq n)$ has $(\mathbf{P})$;

(ii) Every monic polynomial $P(X) \in K[X]$ of degree $t(2 \leq t \leq n)$ has a root in the field $K$.

Proof. (i) $\Rightarrow$ (ii) Let $t_{1}$ be an integer with $2 \leq t_{1} \leq n$. Let $P_{1}(X)=X^{t_{1}}+$ $a_{t_{1}-1} X^{t_{1}-1}+\cdots+a_{1} X+a_{0} \in K[X]$ be a monic polynomial of degree $t_{1}$. Using Remark 2.5, there exists an endomorphism $T_{1}$ of $K^{t_{1}}$ such that the characteristic polynomial of $T_{1}$ is $(-1)^{t_{1}} P_{1}(X)$. By Theorem 2.6, $P_{1}(X)=P_{2}(X) Q_{2}(X)$ where $P_{2}(X), Q_{2}(X) \in K[X]$ with $1 \leq t_{2}=\operatorname{deg}\left(P_{2}(X)\right)<t_{1}$. Repeating this procedure, 
we show that $P_{1}(X)=P(X) Q(X)$ where $P(X), Q(X) \in K[X]$ and $\operatorname{deg}(P(X))=1$. This shows that $P_{1}(X)$ has a root in $K$.

(ii) $\Rightarrow$ (i) Let $V$ be a nonzero $K$-vector space with $2 \leq \operatorname{dim}(V)=t \leq n$ and let $T$ be an automorphism of $V$. By hypothesis, the characteristic polynomial $P_{T}(X)$ of $T$ has a root $\lambda$ in $K$. Therefore there exists a nonzero $u \in V$ such that $T(u)=\lambda u$. Let $W=K u$ be the subspace of $V$ generated by $\{u\}$. It is clear that $T(W) \subseteq W$. Note that $W \neq 0$ and $W \neq V$. So $V$ has $(\mathbf{P})$.

Next, we determine semisimple modules satisfying $(\mathbf{P})$. Recall that a module $M$ is called homogeneous semisimple if it is generated by a single simple module; that is, $M$ is a direct sum of simple modules which are isomorphic to each other.

Example 2.10. Consider the semisimple $\mathbb{Z}$-module $M=(\mathbb{Z} / 3 \mathbb{Z} \oplus \mathbb{Z} / 3 \mathbb{Z}) \oplus \mathbb{Z} / 2 \mathbb{Z}$. Note that $H o m_{\mathbb{Z}}(\mathbb{Z} / 3 \mathbb{Z} \oplus \mathbb{Z} / 3 \mathbb{Z}, \mathbb{Z} / 2 \mathbb{Z})=0$. Then $N=(\mathbb{Z} / 3 \mathbb{Z} \oplus \mathbb{Z} / 3 \mathbb{Z}) \oplus 0$ is a fully invariant submodule of $M$ by [6, Lemma 1.9]. It follows that $M$ has $(\mathbf{P})$. In the same manner we can see that every semisimple module which is not homogeneous has $(\mathbf{P})$.

Proposition 2.11. Let $I$ be an ideal of a commutative ring $R$ and let $M$ be an $R / I$-module. Then the $R$-module ${ }_{R} M$ has $(\mathbf{P})$ if and only if the $R / I$-module ${ }_{R / I} M$ has $(\mathbf{P})$.

Proof. Let $M$ be a nonsimple $R / I$-module. Then $M$ is an $R$-module and the lattices of $R$-submodules and $R / I$-submodules of $M$ coincide. Moreover, any group endomorphism of $M$ is an $R$-endomorphism of $M$ if and only if it is an $R / I$ endomorphism of $M$. The result follows.

Proposition 2.12. Every infinitely generated semisimple module has $(\mathbf{P})$.

Proof. Let $M$ be an infinitely generated semisimple module. By Example 2.10, there is no loss of generality in assuming that $M$ is homogeneous semisimple. Therefore $M \cong(R / \mathfrak{m})^{(\Lambda)}$ for some maximal ideal $\mathfrak{m}$ of $R$ and an infinite index set $\Lambda$. Then $M$ can be viewed as an $R / \mathfrak{m}$-module. By Proposition 2.4 , the $R / \mathfrak{m}$-module $M$ has $(\mathbf{P})$. Thus ${ }_{R} M$ satisfies $(\mathbf{P})$ by Proposition 2.11 .

In the following proposition, we characterize finitely generated homogeneous semisimple modules which have $(\mathbf{P})$.

Proposition 2.13. Let $M$ be a homogeneous semisimple $R$-module such that $M \cong$ $(R / \mathfrak{m})^{n}$ for some maximal ideal $\mathfrak{m}$ of $R$ and some positive integer $n \geq 2$. Let $K=R / \mathfrak{m}$. Then the following are equivalent: 
(i) $M$ has $(\mathbf{P})$ as an $R$-module;

(ii) $M$ has $(\mathbf{P})$ as a $K$-module;

(iii) Every monic polynomial $P(X) \in K[X]$ of degree $n$ is reducible.

Proof. This follows from Theorem 2.6 and Proposition 2.11.

The next corollary follows easily from Proposition 2.13 .

Corollary 2.14. Let a module $M=S_{1} \oplus S_{2}$ be a direct sum of two simple submodules $S_{1}$ and $S_{2}$ such that $S_{1} \cong S_{2} \cong R / \mathfrak{m}$ for some maximal ideal $\mathfrak{m}$ of $R$. Then the following are equivalent:

(i) $M$ has $(\mathbf{P})$;

(ii) Every monic polynomial $P(X) \in K[X]$ of degree 2 has a root in the field $K=R / \mathfrak{m}$.

A direct summand of a module having $(\mathbf{P})$ may not have $(\mathbf{P})$, in general, as shown below.

Example 2.15. Consider the $\mathbb{Z}$-modules $M_{1}=\mathbb{Z} / 2 \mathbb{Z} \oplus \mathbb{Z} / 2 \mathbb{Z}, M_{2}=\mathbb{Z} / 3 \mathbb{Z} \oplus \mathbb{Z} / 3 \mathbb{Z}$ and $M=M_{1} \oplus M_{2}$.

(i) Let $K_{2}=\mathbb{Z} / 2 \mathbb{Z}$ and let the polynomial $P_{1}(X)=X^{2}-X+1 \in K_{2}[X]$. It is clear that $P_{1}(X)$ does not have a root in $K_{2}$. Thus $M_{1}$ does not have $(\mathbf{P})$ by Corollary 2.14 .

(ii) Consider the polynomial $P_{2}(X)=X^{2}+X+2 \in K_{3}[X]$, where $K_{3}=\mathbb{Z} / 3 \mathbb{Z}$. It is easy to check that $P_{2}(X)$ does not have a root in $K_{3}$. From Corollary 2.14, it follows that the module $M_{2}$ does not have $(\mathbf{P})$.

(iii) From Example 2.10, we conclude that the module $M$ has $(\mathbf{P})$. Also, note that both $M_{1}^{(\mathbb{N})}$ and $M_{2}^{(\mathbb{N})}$ have $(\mathbf{P})$ by Proposition 2.12 .

The next result is a direct consequence of Corollary 2.8 and Proposition 2.13.

Corollary 2.16. Let $\mathfrak{m}$ be a maximal ideal of a commutative ring $R$ such that $R / \mathfrak{m}$ is a finite field (for instance, $R$ can be the ring of integers $\mathbb{Z}$ and $\mathfrak{m}=p \mathbb{Z}$ for some prime number $p)$. Then for any positive integer $n \geq 2$, the $R$-module $M=(R / \mathfrak{m})^{n}$ does not have $(\mathbf{P})$.

The next proposition provides more examples of modules having $(\mathbf{P})$ over a commutative ring.

Proposition 2.17. Let $R$ be a commutative ring. Let $M$ be an $R$-module which is not semisimple such that $\operatorname{Rad}(M) \neq M$. Then $M$ has a nonzero proper submodule $N$ that is fully invariant in $M$. In particular, $M$ has $(\mathbf{P})$. 
Proof. Let $\Omega$ denote the set of all maximal ideals of $R$. It is well known that $\operatorname{Rad}(M)=\bigcap_{\mathfrak{m} \in \Omega} M \mathfrak{m}$. Note that $M \mathfrak{m} \neq 0$ for every $\mathfrak{m} \in \Omega$, since otherwise $M$ will be semisimple. In addition, since $\operatorname{Rad}(M) \neq M$, there exists a maximal ideal $\mathfrak{m}_{0}$ of $R$ such that $M \mathfrak{m}_{0} \neq M$. Take $N=M \mathfrak{m}_{0}$. It is easily seen that $N$ is fully invariant in $M$.

Using Example 2.10 and Proposition 2.17, we get the following result.

Corollary 2.18. Let $M$ be a nonzero finitely generated module. If $M$ is not homogeneous semisimple, then $M$ has $(\mathbf{P})$.

Next, we determine all abelian groups which have $(\mathbf{P})$. Let $\mathbb{Q}$ denote the field of rational numbers.

Proposition 2.19. Every direct sum of copies of the $\mathbb{Z}$-module $\mathbb{Q}$ has $(\mathbf{P})$.

Proof. Case 1: Assume that $M=\mathbb{Q}^{(I)}$ where $I$ is an infinite index set. Notice that $M$ has a structure of a $\mathbb{Q}$-module defined by the following operation: given $x \in M, r \in \mathbb{Z}$ and $0 \neq s \in \mathbb{Z}$, we put $(r / s) x=r x^{\prime}$ with $x^{\prime}$ is the unique element of $M$ which satisfies $x=s x^{\prime}$. Note that $x^{\prime}$ exists and is unique because $M$ is a divisible torsion-free $\mathbb{Z}$-module. It is easily seen that $\operatorname{End}_{\mathbb{Z}}(M)=\operatorname{End}_{\mathbb{Q}}(M)$. Also, it is clear that every $\mathbb{Q}$-submodule of $M$ is a $\mathbb{Z}$-submodule of $M$. Applying Proposition 2.4, it follows that $M$ has $(\mathbf{P})$ as a $\mathbb{Q}$-module and hence also as a $\mathbb{Z}$-module.

Case 2: Assume that $M=M_{1} \oplus M_{2}$ such that $M_{i}=\mathbb{Q}$ for each $i=1,2$. It is well known that for any $\mathbb{Z}$-endomorphism $\varphi$ of $\mathbb{Q}$, there exists a nonzero $q \in \mathbb{Q}$ such that $\varphi(x)=q x$ for all $x \in \mathbb{Q}$. Now let $f$ be a nonzero $\mathbb{Z}$-endomorphism of $M$. So there exist integers $a_{1}, a_{2}, c_{1}$ and $c_{2}$ and nonzero integers $b_{1}, b_{2}, d_{1}$ and $d_{2}$ such that for every $\left(x_{1}, x_{2}\right) \in \mathbb{Q}^{2}$, we have $f\left(\left(x_{1}, x_{2}\right)\right)=\left(\left(a_{1} / b_{1}\right) x_{1}+\left(c_{1} / d_{1}\right) x_{2},\left(a_{2} / b_{2}\right) x_{1}+\right.$ $\left.\left(c_{2} / d_{2}\right) x_{2}\right)$. Let $p$ be a prime integer which does not divide $b_{1} d_{1} b_{2} d_{2}$. Consider the non-trivial $\mathbb{Z}$-submodule $L=\{m / n \mid p$ does not divide $n\}$ of $\mathbb{Q}$. Set $N=N_{1} \oplus N_{2}$ such that $N_{i}=L$ for each $i=1,2$. Then $N$ is a non-trivial submodule of $M$ that is invariant under $f$. This shows that $M$ has $(\mathbf{P})$. In the same manner we can see that every finite direct sum of copies of $\mathbb{Q}$ satisfies $(\mathbf{P})$.

Remark 2.20. Consider the $\mathbb{Z}$-module $M=\mathbb{Q}^{2}$. Let $P(X)=X^{2}-2 \in \mathbb{Q}[X]$. It is clear that $P(X)$ does not have a root in $\mathbb{Q}$. By Corollary 2.14, $M$ considered as a $\mathbb{Q}$-module does not have $(\mathbf{P})$. On the other hand, $M$ viewed as a $\mathbb{Z}$-module satisfies $(\mathbf{P})$ by Proposition 2.19 . 
Theorem 2.21. The following are equivalent for a $\mathbb{Z}$-module $M$ :

(i) $M$ has $(\mathbf{P})$;

(ii) $M$ satisfies any one of the following conditions:

(a) $M$ is not semisimple, or

(b) $M$ is a semisimple module which is infinitely generated or not homogeneous.

Proof. (i) $\Rightarrow$ (ii) From Example 2.10, Proposition 2.12 and Corollary 2.16, it follows that a semisimple $\mathbb{Z}$-module has $(\mathbf{P})$ if and only if it is infinitely generated or not homogeneous. Now assume that $M$ is not semisimple.

Case 1: $\operatorname{Rad}(M) \neq M$. In this case $M$ has $(\mathbf{P})$ by Proposition 2.17.

Case 2: $\operatorname{Rad}(M)=M$ and $\operatorname{Soc}(M) \neq 0$. Since $M$ is not semisimple, we have $\operatorname{Soc}(M) \neq M$. Hence $\operatorname{Soc}(M)$ is a non-trivial fully invariant submodule of $M$. This clearly implies that $M$ has $(\mathbf{P})$.

Case 3: $\operatorname{Rad}(M)=M$ and $\operatorname{Soc}(M)=0$. In this case $M$ is a divisible torsion-free $\mathbb{Z}$-module. Hence $M$ is isomorphic to a direct sum of copies of $\mathbb{Q}$. Therefore $M$ has (P) by Proposition 2.19 .

\section{Rings whose modules satisfy $(\mathbf{P})$}

The aim of this section is to characterize the class of rings $R$ over which every nonzero finitely generated $R$-module $M$ which is not simple satisfies $(\mathbf{P})$. Let $R$ be a commutative ring and consider the following properties:

$\left(\mathbf{P}_{\mathbf{1}}\right)$ : Every nonzero finitely generated $R$-module $M$ which is not simple satisfies $(\mathbf{P})$.

$\left(\mathbf{P}_{\mathbf{2}}\right)$ : Every nonsimple $R$-module $M$ with $\operatorname{Rad}(M) \neq M$ satisfies $(\mathbf{P})$.

$\left(\mathbf{P}_{\mathbf{3}}\right)$ : Every nonzero $R$-module $M$ which is not simple satisfies $(\mathbf{P})$.

Recall that a field $K$ is called an algebraically closed field if any polynomial in $K[X]$ of degree $n \geq 1$ has at least one root in $K$.

Proposition 3.1. Let $K$ be a field. Then the following are equivalent:

(i) $K$ satisfies $\left(\mathbf{P}_{\mathbf{1}}\right)$;

(ii) $K$ satisfies $\left(\mathbf{P}_{\mathbf{2}}\right)$;

(iii) $K$ satisfies $\left(\mathbf{P}_{\mathbf{3}}\right)$;

(iv) $K$ is algebraically closed.

Proof. (iii) $\Rightarrow$ (ii) $\Rightarrow$ (i) are immediate.

(i) $\Rightarrow$ (iv) This follows from Proposition 2.9. 
(iv) $\Rightarrow$ (iii) Let $V$ be a $K$-vector space with $\operatorname{dim}(V) \geq 2$. If $V$ is of infinite dimension, then $V$ has $(\mathbf{P})$ by Proposition 2.4. If $V$ is finite-dimensional, then $V$ has $(\mathbf{P})$ by Theorem 2.6. Therefore $K$ satisfies $\left(\mathbf{P}_{\mathbf{3}}\right)$.

Proposition 3.2. Let $R$ be a commutative ring. If $R$ satisfies $\left(\mathbf{P}_{\mathbf{1}}\right)$ (resp., $\left(\mathbf{P}_{\mathbf{2}}\right)$ or $\left(\mathbf{P}_{\mathbf{3}}\right)$ ), then $R / I$ satisfies $\left(\mathbf{P}_{\mathbf{1}}\right)$ (resp., $\left(\mathbf{P}_{\mathbf{2}}\right)$ or $\left(\mathbf{P}_{\mathbf{3}}\right)$ ) for every ideal $I$ of $R$.

Proof. This follows from Proposition 2.11.

Combining Propositions 3.1 and 3.2, we obtain the following corollary.

Corollary 3.3. Let $R$ be a commutative ring. If $R$ satisfies $\left(\mathbf{P}_{\mathbf{1}}\right)$, then the field $R / \mathfrak{m}$ is algebraically closed for every maximal ideal $\mathfrak{m}$ of $R$.

We call a ring $R \mathfrak{m}$-algebraically closed if $R / \mathfrak{m}$ is an algebraically closed field for all maximal ideals $\mathfrak{m}$ of $R$.

Theorem 3.4. The following conditions are equivalent for a commutative ring $R$ :

(i) $R$ satisfies $\left(\mathbf{P}_{\mathbf{1}}\right)$;

(ii) $R$ satisfies $\left(\mathbf{P}_{\mathbf{2}}\right)$;

(iii) $R$ is an $\mathfrak{m}$-algebraically closed ring.

Proof. (ii) $\Rightarrow$ (i) This is clear.

(i) $\Rightarrow$ (iii) This follows from Corollary 3.3.

(iii) $\Rightarrow$ (ii) Using Example 2.10 and Proposition 2.17, we only need to show that every semisimple homogeneous $R$-module which is not simple satisfies $(\mathbf{P})$. Let $M$ be a nonzero semisimple homogeneous $R$-module such that $M$ is not simple. Note that $M \cong(R / \mathfrak{m})^{(\Lambda)}$ for some maximal ideal $\mathfrak{m}$ of $R$ and some index set $\Lambda$. Hence $M$ can be considered as an $R / \mathfrak{m}$-module. Since $R / \mathfrak{m}$ is algebraically closed, it follows that the $R / \mathfrak{m}$-module $R / \mathfrak{m} M$ satisfies $(\mathbf{P})$ by Proposition 3.1. Therefore the $R$-module ${ }_{R} M$ satisfies $(\mathbf{P})$ by Proposition 2.11. This proves the theorem.

Remark 3.5. It is well known that a finite field could not be algebraically closed. From Theorem 3.4, it follows that a finite ring could not satisfy $\left(\mathbf{P}_{\mathbf{1}}\right)$.

Next, we exhibit some examples of rings satisfying properties $\left(\mathbf{P}_{\mathbf{1}}\right)$ and $\left(\mathbf{P}_{\mathbf{2}}\right)$.

Example 3.6. (i) Let $K_{1}, K_{2}, \ldots, K_{n}$ be algebraically closed fields. Applying Theorem 3.4, we see that the ring $R=K_{1} \times K_{2} \times \cdots \times K_{n}$ satisfies $\left(\mathbf{P}_{\mathbf{2}}\right)$.

(ii) Let $K$ be an algebraically closed field and let $R=K\left[X_{1}, \ldots, X_{n}\right]$. It is well known (see Hilbert's Nullstellensatz) that the maximal ideals of the ring $R$ are the ideals $\left(X_{1}-a_{1}, X_{2}-a_{2}, \ldots, X_{n}-a_{n}\right)$, where $a_{1}, a_{2}, \ldots, a_{n} \in K$. Moreover, for any $a_{1}, a_{2}, \ldots, a_{n} \in K,\left(X_{1}-a_{1}, X_{2}-a_{2}, \ldots, X_{n}-a_{n}\right)$ is the kernel of the epimorphism 


$$
\varphi: R \rightarrow K \text { defined by } f \mapsto f\left(a_{1}, a_{2}, \ldots, a_{n}\right) .
$$

Thus $R /\left(X_{1}-a_{1}, X_{2}-a_{2}, \ldots, X_{n}-a_{n}\right)$ is isomorphic to $K$. This implies that $R /\left(X_{1}-a_{1}, X_{2}-a_{2}, \ldots, X_{n}-a_{n}\right)$ is an algebraically closed field. Hence $R$ satisfies $\left(\mathbf{P}_{2}\right)$ by Theorem 3.4. Note that the ring $R$ has infinitely many maximal ideals.

(iii) Let $K$ be a field and let $R$ be a subring of $K$. Recall that $R$ is called a valuation ring of $K$ if, for any $0 \neq x \in K$, either $x \in R$ or $x^{-1} \in R$. Note that every valuation ring of $K$ is a local ring.

Now assume that $K$ is an algebraically closed field and let $R$ be a valuation ring of $K$. It is well known that the residue field of $R$ is also algebraically closed. From Theorem 3.4, we see that the ring $R$ satisfies $\left(\mathbf{P}_{\mathbf{2}}\right)$.

Proposition 3.7. A finite product $R=\prod_{i=1}^{n} R_{i}(n \geq 2)$ of rings satisfies $\left(\mathbf{P}_{\mathbf{2}}\right)$ if and only if so is each $R_{i}(1 \leq i \leq n)$.

Proof. There is no loss of generality in assuming that $n=2$. The necessity follows from Proposition 3.2. Conversely, let $\mathfrak{m}$ be a maximal ideal of $R$. Then $\mathfrak{m}=\mathfrak{m}_{1} \times R_{2}$ or $\mathfrak{m}=R_{1} \times \mathfrak{m}_{2}$, where $\mathfrak{m}_{i}(i \in\{1,2\})$ is a maximal ideal of $R_{i}$. Hence $R / \mathfrak{m} \cong R_{1} / \mathfrak{m}_{1}$ (as fields) or $R / \mathfrak{m} \cong R_{2} / \mathfrak{m}_{2}$ (as fields). Using Theorem 3.4 twice, we conclude that $R / \mathfrak{m}$ is an algebraically closed field and hence the ring $R$ satisfies $\left(\mathbf{P}_{\mathbf{2}}\right)$.

Acknowledgement. The authors would like to thank the referee for helpful comments that improved the paper and for providing them with Example 2.7.

\section{References}

[1] N. Aronszajn and K. T. Smith, Invariant subspaces of completely continuous operators, Ann. of Math., 60(2) (1954), 345-350.

[2] A. R. Bernstein and A. Robinson, Solution of an invariant subspace problem of K. T. Smith and P. R. Halmos, Pacific J. Math., 16(3) (1966), 421-431.

[3] R. Lidl and H. Niederreiter, Introduction to Finite Fields and their Applications, Revised edition, The Press Syndicate of the University of Cambridge, Cambridge University Press, Cambridge, 1994.

[4] M. Liu, The invariant subspace problem and its main developments, Int. J. Open Problems Compt. Math., 3(5) (2010), 88-97.

[5] V. I. Lomonosov, Invariant subspaces for the family of operators which commute with a completely continuous operator, Funct. Anal. Appl., 7(3) (1973), 213-214.

[6] A. Ç. Özcan, A. Harmanci and P. F. Smith, Duo modules, Glasg. Math. J., 48(3) (2006), 533-545. 
Mohamed Benslimane and Hanane EL Cuera

Department of Mathematics

Faculty of Sciences

Abdelmalek Essaâdi University

B.P. 2121 Tetouan, Morocco

e-mails: med.bens@gmail.com (M. Benslimane)

hanane.cuera@gmail.com (H. EL Cuera)

Rachid Tribak (Corresponding Author)

Centre Régional des Métiers de l'Education et de la Formation (CRMEF-TTH)-Tanger

Avenue My Abdelaziz, Souani, B.P. 3117, Tangier, Morocco

e-mail: tribak12@yahoo.com 